\title{
Calibration of low-frequency radio telescopes using the galactic background radiation
}

\author{
G. A. Dulk ${ }^{1}$, W. C. Erickson ${ }^{2}$, R. Manning ${ }^{1}$, and J.-L. Bougeret ${ }^{1}$ \\ 1 NRS-URA 264, Département de Recherche Spatiale, Observatoire de Paris, 92195 Meudon, France \\ ${ }^{2}$ Department of Physics, University of Tasmania, Hobart, Tas 7001, Australia
}

Received 28 February 2000 / Accepted 28 September 2000

\begin{abstract}
We consider the calibration of flux densities of radio bursts from decametric to kilometric wavelengths using ground-based and space-based data. The method we derive is applicable to low-frequency radio telescopes where galactic background radiation is the principal contribution to system temperature. It can be particularly useful for telescopes of low angular resolution observing spectra of radio bursts from the Sun and the planets because absolute calibration of these telescopes is very difficult with conventional techniques. Here we apply the method to observations from about 7 to $47 \mathrm{MHz}$ that were made on the ground with the Bruny Island Radio Spectrometer located in Tasmania, Australia, and those from about $20 \mathrm{kHz}$ to $13.8 \mathrm{MHz}$ were made with the radio experiment WAVES on the WIND spacecraft. The spectrum of the galactic background radiation from $<1$ to $>30 \mathrm{MHz}$ has been carefully measured with low-resolution telescopes, starting more than a decade ago. We use this known spectrum to calibrate both BIRS and WAVES on an absolute scale. The accuracy we achieve is about a factor of two, whereas the flux densities of solar and planetary radio sources vary by many orders of magnitude. Our method permits inter-calibration of ground-based and space-based observations, and allows corrections to be made for instrumental uncertainties on both radio experiments. In addition, on the ground, it allows the spectra to be corrected for ionospheric absorption and partial ground reflections. As an application we show the spectrum of a solar type III burst observed from $47 \mathrm{MHz}$ to $20 \mathrm{kHz}$. Its flux density was largest, $S \approx 10^{-17} \mathrm{~W} \mathrm{~m}^{-2} \mathrm{~Hz}^{-1}$, at about $3 \mathrm{MHz}$, while at $60 \mathrm{kHz}$ and at $47 \mathrm{MHz}$ it was lower by a factor of about 300 .
\end{abstract}

Key words. radio telescopes - calibration - galactic background radiation - solar type III bursts

\section{Introduction}

The flux density of low-frequency radio bursts has rarely been observed over a large range of frequency. Up to now there has always been a large gap in the frequency coverage: typically space-based observations were at frequencies lower than 1-2 MHz, and ground-based observations were at frequencies higher than 20-30 MHz. However, it was known that solar type III bursts observed at metric wavelengths often disappear in the decametric range. Other bursts appear at decametric wavelengths and may or may not continue to the low-frequency limit of most groundbased spectrographs of about $20 \mathrm{MHz}$. On the other hand, many bursts observed in space commence below $\sim 10 \mathrm{MHz}$, sometimes below $1 \mathrm{MHz}$ (e.g. review by Dulk 2000).

In the very few studies of bursts that continue from decameter to kilometer wavelengths, Weber (1978) and Steinberg et al. (1984) showed (but with a large gap in frequency coverage) that the spectra of flux density and brightness temperature of type III bursts typically increase with decreasing frequency down to about $1 \mathrm{MHz}$,

Send offprint requests to: G. A. Dulk, e-mail: dulk@obspm.fr with a maximum that is usually between 0.5 and $5 \mathrm{MHz}$, and a decrease at yet lower frequencies. Occasionally the flux density near $1 \mathrm{MHz}$ can attain very high values, up to $10^{-14} \mathrm{~W} \mathrm{~m}^{-2} \mathrm{~Hz}^{-1}$ (Weber 1978; Leblanc et al. 2000).

Only recently have two radio telescopes been put in operation that can provide continuous spectra from decametric to kilometric wavelengths with sufficient overlap to insure that the comparison is valid. These are the Bruny Island Radio Spectrometer (BIRS) located near Hobart, Tasmania, Australia (Erickson 1997), and the WAVES experiment on the WIND spacecraft (Bougeret et al. 1995). BIRS covers the frequency range of 3 to $47 \mathrm{MHz}$ and WAVES covers from $<0.01$ to $13.8 \mathrm{MHz}$. Tasmania is an excellent place to observe radio emissions at low frequencies because there is little local interference and the ionosphere above it is of unusually low electron density; observations are possible down to $2-3 \mathrm{MHz}$ at night, and 5-10 $\mathrm{MHz}$ during the day, with conditions being best near the minimum of the sunspot cycle.

Combining BIRS and WAVES observations of radio bursts, we have continuous coverage from about $20-30 \mathrm{kHz}$ 
(cutoff by the plasma frequency at the WIND spacecraft) to $47 \mathrm{MHz}$, with an overlap from the cutoff by the ionosphere in Tasmania at 5-10 MHz to the highest frequency of WAVES of 13.8 MHz. However, when we first compared the flux densities of type III bursts in the overlap region they were grossly discrepant (see Sect. 3). In ensuing discussions we recognized that both instruments observe the galactic background radiation and that this galactic emission can be used as a common calibration source because its spectrum is well known (Cane 1979). In addition to insuring good flux density calibrations for both instruments, the galactic emission is useful for making corrections for antenna characteristics and ionospheric effects.

The method of calibration we develop, using the galactic background radiation as the calibration source, can be usefully applied to radio telescopes at $\lesssim 100 \mathrm{MHz}$ where the system temperature is dominated by the galactic radiation, and is especially useful for telescopes of low spatial resolution that are otherwise difficult to calibrate. Here the method is applied to a solar type III burst in the frequency range $20 \mathrm{kHz}$ to $47 \mathrm{MHz}$. The spectrum has its maximum at about $3 \mathrm{MHz}$, with the peak value being $10^{-17} \mathrm{~W} \mathrm{~m}^{-2} \mathrm{~Hz}^{-1}$.

In Sect. 2 we describe briefly the BIRS and WAVES radio instruments, in Sect. 3 we develop the method to calibrate the two instruments using the galactic background radiation as a common calibration source, in Sect. 4 we apply the method to a solar type III burst observed over the entire frequency range, and give concluding remarks.

\section{The radio instruments}

\subsection{BIRS: The Bruny Island Radio Spectrometer}

BIRS is located on Bruny Island, about $50 \mathrm{~km}$ south of Hobart, Tasmania in Australia at latitude $\mathrm{S} 42.37^{\circ}$, longitude E147.218 ${ }^{\circ}$. The instrument has been described in detail by Erickson (1997). Here we summarize its characteristics that are important for this study.

The antenna is a 25-element log-periodic structure with linearly polarized dipoles. The supporting mast, $20.4 \mathrm{~m}$ high, holds the vertex of the triangular antenna plane that is directed to the intersection of the meridian and the celestial equator. The dipoles vary in length from $3.06 \mathrm{~m}$ to $50.0 \mathrm{~m}$, giving an antenna gain of approximately $6 \mathrm{db}$ between $\approx 3$ and $47 \mathrm{MHz}$. The beamwidth in the meridian plane is more than $100^{\circ}$, while that in the $\mathrm{E}-\mathrm{W}$ direction is about $90^{\circ}$. The system is operated from 2200 UT to 0630 UT each day, but observations of the Sun with good sensitivity, i.e. with the Sun within the half-power beamwidth, occur only for about 6 hours per day.

The radio spectrum below about $25 \mathrm{MHz}$ is used for intercontinental communication and is crowded with strong, narrow band signals. The spectrometer is controlled by a small computer that is programmed to detect the spaces between these signals and to use them for the solar observations. The system automatically adapts itself to the ever changing signal spectrum in order to obtain relatively interference-free observations. The remaining interference that cannot be avoided by the system is later excised by an off-line program. This program also excises short-period, wide-band spikes from both local and tropical lightening strokes. These procedures result in solar observations that are nearly interference-free.

Aside from its adaptive interference avoidance features, the system is quite straight-forward. The antenna signals are first amplified by a wideband, low noise amplifier and then transmitted to the laboratory via coaxial cable. After further amplification in the laboratory, the signals pass through a network that attenuates the lower part of the spectrum, below $25 \mathrm{MHz}$, by $20 \mathrm{~dB}$. After further amplification, the signal is presented to a computercontrolled, commercial spectrum analyzer. At all frequencies the system noise is dominated by the galactic background. The $20 \mathrm{~dB}$ attenuation reduces the otherwise very high background levels in the lower portion of the spectral range and reduces the dynamic range requirements for the spectrum analyzer. The spectrometer has a logarithmic response and a dynamic range up to about $70 \mathrm{~dB}$ above the galactic background. The computer selects 128 fairly equally-spaced, interference-free frequencies, one in each $344 \mathrm{kHz}$ band across the 3 to $47 \mathrm{MHz}$ spectrum, and samples these frequencies every $3 \mathrm{~s}$. The instantaneous bandwidth of the spectrometer is $10 \mathrm{kHz}$. The data are recorded on disk each $30 \mathrm{~min}$ and are transferred to the internet (fourier.phys.utas.edu.au/birs) at the end of each day's observations.

\subsection{WAVES: The radio experiment on the WIND spacecraft}

The WIND spacecraft is placed in the solar wind, often near the L1 Lagrangian point about 200 Earth radii in the sunward direction of Earth. The WAVES experiment (Bougeret et al. 1995) includes a three-receiver radio spectrometer that operates from a few $\mathrm{kHz}$ to $13.8 \mathrm{MHz}$. The receivers are fed from three dipole antennas. The $X$-antenna, $2 \times 50 \mathrm{~m}$ long and the $Y$-antenna, $2 \times 7.5 \mathrm{~m}$ long, are in the spin plane of the spacecraft which is aligned with the ecliptic plane. The spacecraft rotates once per $3 \mathrm{~s}$. The $Z$-antenna is aligned with the spin axis of the spacecraft; its length was $2 \times 4.3 \mathrm{~m}$ from launch in November 1994 until 20 Nov. 1996, and then it was lengthened to $2 \times 5.3 \mathrm{~m}$.

The signals from the antennas are fed to three receivers: RAD2 ( $Y$ and/or $Z$ antennas) for the high frequency range of 1.075 to $13.8 \mathrm{MHz}$, RAD1 ( $X$ and/or $Z$ antennas) for the lower frequency range of 0.02 to $1.04 \mathrm{MHz}$, and the thermal noise receiver TNR (usually $X$ antenna) for the lowest frequency range of 0.004 to $0.256 \mathrm{MHz}$. In this paper we make little use of the TNR except to determine the local plasma frequency at WIND through the presence of the quasi-thermal plasma line. 
The RAD1 and RAD2 receivers can be operated in several modes. For example, when RAD1 utilizes the $X$ and $Z$ antenna signals in a summation mode, the direction of the source centroid can be determined in two dimensions and the source size can be estimated. Similar direction finding is possible with RAD2 with the $Y$ and $Z$ antennas.

The number of frequencies sampled by the receivers is flexible. In one mode of RAD2, 256 equally-spaced frequencies are sampled every $16 \mathrm{~s}$. In another mode a list of frequencies is sampled more frequently (e.g. 16 frequencies, each sampled once per second), or with higher frequencies being sampled more frequently than the lower ones where the flux varies less rapidly. Normally the sampling is done in a sequence where 8 or 12 samples are taken at a given frequency within half of the $3 \mathrm{~s}$ spin period to permit direction finding.

\section{Calibration of BIRS and WAVES using the galactic background radiation}

The first spectra of solar type III bursts obtained simultaneously at BIRS and WAVES used a standard, noisetube calibration method at BIRS, the "standard method" described below for RAD1, and preliminary, pre-launch measurements for RAD2. The flux densities in the overlap range between about 7 and $13.8 \mathrm{MHz}$ disagreed significantly (the RAD2 values being higher than those of BIRS by more than a factor of 10 for reasons that are still being investigated). As mentioned in the introduction, we recognized that both instruments observe the galactic background and that this galactic emission could be used as a common calibration source. The galactic radiation is unpolarized and is the dominant source of noise over much of the frequency range from less than $1 \mathrm{MHz}$ to more than $100 \mathrm{MHz}$. The galactic background is also useful as a calibration source because the solar and galactic signals follow the same paths through the antennas and receivers, causing many sources of error to cancel. In particular, ohmic losses in the antenna systems cancel, allowing us to consider the antennas to be lossless.

\subsection{The galactic background radiation}

The radio spectrum of the galactic background radiation has been studied by many observers. For example, Cane (1979) observed at several frequencies in the northern and southern hemispheres with identical equipment, half-wave dipoles over a ground screen. Thus the observations were made with an angular resolution comparable to those of BIRS and WAVES, which is important because the response of an antenna to the brightness distribution of the galactic background radiation depends on angular resolution and radio frequency.

Cane (1979) then combined her results with about 100 independent measurements by $\gtrsim 20$ observers, both space-based (e.g. Alexander et al. 1969; Brown 1973; Novaco \& Brown 1978) and ground-based (e.g. Yates \& Wielebinski 1966). The resulting spectrum, for high galactic latitudes, is probably the most reliable estimate of galactic background levels in the $1-100 \mathrm{MHz}$ range. Although these data are more than 20 years old, they agree well with modern ground-based measurements. For example, following careful measurement of the BIRS antenna characteristics, nightime galactic spectra when ionospheric absorption is low are in accord with the spectrum of Cane.

For the direction of the galactic poles, in particular the south galactic pole (SGP), the resulting spectrum of specific intensity in units of $\mathrm{W} \mathrm{m} \mathrm{m}^{-2} \mathrm{~Hz}^{-1} \mathrm{sr}^{-1}$ is well described by the equation:

$I_{\nu}=I_{\mathrm{g}} \nu^{-0.52} \frac{1-\exp [-\tau(\nu)]}{\tau(\nu)}+I_{\mathrm{eg}} \nu^{-0.80} \exp [-\tau(\nu)]$

where $\nu$ is in $\mathrm{MHz}$, the first and second terms are the galactic and extragalactic contributions respectively, and the opacity in the polar direction is $\tau(\nu)$. The values of the parameters are:

$I_{\mathrm{g}}=2.4810^{-20}, \quad I_{\mathrm{eg}}=1.0610^{-20}, \quad \tau(\nu)=5.0 \nu^{-2.1}$.

Cane $(1977,1978)$ also produced 10 and $30 \mathrm{MHz}$ maps of apparent brightness temperature, $T_{\mathrm{b}}$, for essentially the whole sky. We note that $I_{\nu}$ and $T_{\mathrm{b}}$ are related by the Rayleigh-Jeans Law:

$k T_{\mathrm{b}}=I_{\nu} c^{2} / 2 \nu^{2}$.

Over large solid angles away from the galactic plane the brightness temperature is fairly constant and Eq. (1) is applicable. However, the brightness temperature rises by a factor of five to ten near the galactic plane and displays much more structure in angle. It would be rather difficult to accurately predict the response of most antennas to galactic emission near the plane, but fortunately the high brightness structures subtend a much smaller solid angle than the mid-to-high latitude regions; thus the total response of a low-gain antenna can be estimated with reasonable accuracy, i.e. to a factor of 2 or better.

\subsection{Application to BIRS}

The beam solid angle of the BIRS antenna is $\Omega_{\text {beam }} \approx$ $2.8 \mathrm{sr}$. The response of the antenna to the galactic background varies with local sidereal time (LST), and at $\mathrm{LST}=00^{\mathrm{h}} 50^{\mathrm{m}}$ the antenna's response pattern is aligned in Right Ascension with the SGP but centered some $27^{\circ}$ (about one-quarter beamwidth) north of it. Nevertheless, the area of sky within the antenna's response pattern is basically the south polar cap and the antenna temperature should equal the brightness temperature as given by Eqs. (1) and (3). This provides a temperature calibration at each frequency. The receiver noise temperature is negligible compared with the galactic temperature.

The observed diurnal variation of antenna temperature at 10 and $30 \mathrm{MHz}$ is also in reasonable agreement with that which would be predicted from Cane's maps. 


\subsubsection{Procedure}

The calibration of the BIRS data involves several steps. First, the spectrum of the south galactic polar region is observed at a local sidereal time of $00^{\mathrm{h}} 50^{\mathrm{m}}$ when the SGP is most nearly centered in the beam of the antenna. This observation is made late at night when ionospheric absorption is low. The antenna temperature is compared with that of a thermionic noise source signal that is introduced at the antenna terminals. These data are used to calibrate the noise source, which is found to be stable over time periods of months and years. Frequent system calibrations employing this noise source are then used to estimate the antenna temperature generated by a solar burst. As mentioned above, ohmic losses in the antenna are automatically compensated by the use of the galactic background as the calibration reference. However, conversion of antenna temperature to flux density requires a knowledge of the antenna gain in the direction of the source. This antenna pattern was estimated from curves in standard references and also was modeled at the Radiophysics Laboratory, Sydney. The main lobe patterns determined in these fashions were identical. On the other hand, the response pattern of the antenna is not known well enough for reliable flux densities to be derived at angles much beyond the half-power points, $45^{\circ}$ east or west of the local meridiam.

Finally, the ionospheric attenuation of the solar signal must be estimated. This is done by observing the galactic background spectrum at times immediately adjacent to the solar burst. The observed galactic spectrum is then compared with a catalogued galactic spectrum that was observed at the same local sidereal time but at a different time of the year, i.e. late at night under conditions of minimal ionospheric absorption. The difference between the observed and the catalogued galactic spectra is used to correct the solar burst spectrum for ionospheric attenuation. This correction is only approximate because the observed background spectrum is affected by an average absorption over the antenna beam while the solar spectrum is affected only by absorption in the specific direction of the sun.

\subsection{Application to WAVES}

The WAVES antennas are dipoles, short dipoles in much of their respective frequency ranges. Their response pattern is well known and their beam solid angle is $\Omega_{\text {beam }}=$ $8 \pi / 3=8.4 \mathrm{sr}$, which is larger than that of the BIRS antenna and those used by Cane. Roughly, this pattern encompasses a band of sky $84^{\circ}$ wide centered on the plane perpendicular to the dipole.

As part of the calibration scheme we need to know the response of the antenna to the distribution of galactic brightness. This response is $S_{\text {beam }}$, the "flux density per beam", i.e. the distribution of specific intensity of the source weighted by the antenna response function.
Consistent with Kraus, (1966, Eqs. (3-19), (3-21)), we define it here to be:

$S_{\text {beam }}=\int_{4 \pi} \mathrm{d} \Omega I_{\nu}(\Omega) A(\Omega) / A_{0} \quad \mathrm{~W} \mathrm{~m}^{-2} \mathrm{~Hz}^{-1}$

where $I_{\nu}(\Omega)$ is the specific intensity of the source in direction $\Omega$, and where $A(\Omega)\left(\mathrm{m}^{2}\right)$ is the effective collecting area of the antenna in direction $\Omega$ and $A_{0}$ is its maximum value.

Note that $S_{\text {beam }}$ is related to the spectral power available from the antenna, $w(\nu)$, or the antenna temperature, $T_{\mathrm{a}}$, where

$w(\nu)=k T_{\mathrm{a}}=S_{\text {beam }} A_{0} / 2 \quad \mathrm{~W} \mathrm{~Hz}^{-1}$.

It is useful to consider the specific intensity of the galactic background radiation to be composed of two components: an isotropic component on which is superimposed an enhancement that is concentrated near the galactic plane.

The isotropic component describes the brightness of

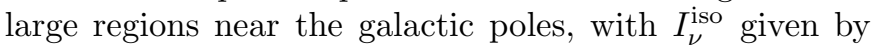
Eqs. (1) and (2). The flux density per beam of the isotropic component is

$S_{\text {beam }}^{\text {iso }}=I_{\nu}^{\text {iso }} / A_{0} \int_{4 \pi} \mathrm{d} \Omega A(\Omega) \quad \mathrm{W} \mathrm{m}^{-2} \mathrm{~Hz}^{-1}$.

A general relation is

$\int_{4 \pi} \mathrm{d} \Omega A(\Omega)=\lambda^{2}=A_{0} \times \Omega_{\text {beam }} \quad \mathrm{m}^{2} \mathrm{sr}$.

Therefore, the flux density per beam for a short dipole antenna $\left(\Omega_{\text {beam }}=8 \pi / 3 \mathrm{sr}, A_{0}=\lambda^{2} \times 3 / 8 \pi\right)$ due to the isotropic galactic background radiation $I_{\nu}^{\text {iso }}$ described by Eq. (1) is

$S_{\text {beam }}^{\text {iso }}=I_{\nu}^{\text {iso }} \Omega_{\text {beam }}=8 \pi / 3 I_{\nu}^{\text {iso }} \quad \mathrm{W} \mathrm{m}^{-2} \mathrm{~Hz}^{-1}$.

We now add the response of the short dipole antenna to the enhanced brightness of the galactic plane. The $Z$ dipole is the simplest to consider since it is aligned with the spin axis of the spacecraft and its response pattern is stationary on the sky. The maximum of its response pattern is in the ecliptic plane which passes only about $5^{\circ}$ north of the galactic center. Using Cane's $10 \mathrm{MHz}$ map, the average intensity within the beam solid angle of this dipole is estimated to be $1.3 \times I_{\nu}^{\text {iso }}$. Equation (8) multiplied by 1.3 is then used to provide a calibration for the $Z$-dipole at $10 \mathrm{MHz}$.

The $X$ and $Y$-dipoles spin in the ecliptic plane. When they are aligned with an ecliptic longitude of $0^{\circ}$ the maxima of their response patterns pass near the galactic center and we expect them to respond to an average intensity that is again about $1.3 \times I_{\nu}^{\text {iso }}$. However, when they are aligned with a longitude of $90^{\circ}$, they are insensitive to the high intensity region near the galactic center and the average intensity in their beam solid angles should be close to $I_{\nu}^{\text {iso }}$ itself. This is borne out by measurements of the spin modulation of the galactic background by these antennas (R. Manning, private communication) which is found to be about 1.3 at $10 \mathrm{MHz}$. 


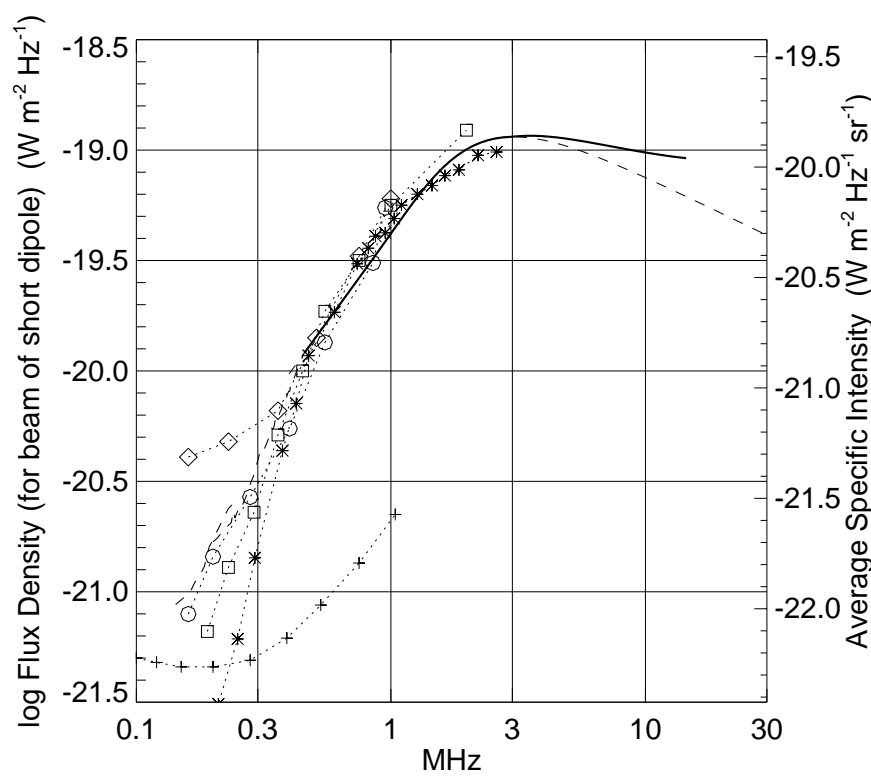

Fig. 1. Left scale: flux density per beam of galactic background radiation for a short dipole in free space in the range 0.1 to $30 \mathrm{MHz}$. Right scale: specific intensity averaged over the $8 \pi / 3 \mathrm{sr}$ beam of a short dipole. The meanings of the thick and dashed lines are explained in the text. The dashed line extension below $0.5 \mathrm{MHz}$ gives the flux density per beam of the short dipole that we derive from WAVES. The dotted lines marked with squares and diamonds show measurements from ISEE-3 (M. Reiner, J.-L. Bougeret, unpublished), the one marked with circles is from Ulysses (S. Hoang, unpublished), that marked with asterisks is the minimum specific intensity from Brown (1973), and the curve with + marks is our present estimate of the receiver noise level of WAVES

Because of free-free absorption in the interstellar medium at frequencies below $10 \mathrm{MHz}$, the galactic plane becomes less bright relative to the poles, and at about $3 \mathrm{MHz}$ the galactic background radiation is essentially isotropic to antennas with low directivity such as dipoles in free space (e.g. Novaco \& Brown 1978). Therefore, we taper the correction factor from 1.3 at $10 \mathrm{MHz}$ to 1.0 at $3 \mathrm{MHz}$.

In Fig. 1, the thick solid line shows the final spectrum, denoted $S_{\text {beam }}^{\text {gal }}$, that we use to calibrate the WAVES observations. The left scale shows it in terms of flux density per beam of a short dipole. The right scale shows it as the average specific intensity, which is lower by the factor $\Omega_{\text {beam }}=8 \pi 3$ of a short dipole. In the range 3 to $13.8 \mathrm{MHz}$ the correction for the galactic plane causes the thick solid line to deviate from the dashed line, which represents the isotropic component of Eq. (1).

The other curves in Fig. 1 show two unpublished measurements of the galactic background intensity by the ISEE-3 spacecraft from 0.2 to $2 \mathrm{MHz}$, one by Ulysses to $1 \mathrm{MHz}$, and the "minimum values" measured by the IMP-6 spacecraft (Brown 1973). Near the bottom is our preliminary measurement of the receiver noise of WAVES. From about 0.5 to $2 \mathrm{MHz}$ the measurements are in good agreement with each other and with the extrapolated galactic spectrum. Below about $0.4 \mathrm{MHz}$ the measurements indicate that the galactic background intensity decreases more rapidly with wavelength than does an extrapolation of Cane's spectrum. Below about $0.15 \mathrm{MHz}$ we show no measurements: the quasi-thermal plasma noise is more and more intense than the galactic background radiation and measurements are more and more uncertain.

\subsection{Procedure}

The WAVES antennas are connected to the receiver terminals through a resistive/capacitive network $X(\nu)$ whose properties were measured prior to launch. The spectral voltage at the receiver input is measured in units of $\mu \mathrm{V} / \mathrm{Hz}^{1 / 2}$. The square of this voltage, $P(\nu)$, is proportional to the spectral power, $w(\nu)$ in $\mathrm{W} \mathrm{Hz}^{-1}$, of Eq. (5). The usual method to convert from the measured $P(\nu)$ in $\mu \mathrm{V}^{2} / \mathrm{Hz}$ to flux density in physical units such as $\mathrm{W} \mathrm{m} \mathrm{m}^{-2} \mathrm{~Hz}^{-1}$ involves $X(\nu), P(\nu)$, and a known constant of proportionality $C$, i.e., $S(\nu)=C \times X(\nu) \times P(\nu) / A_{\text {eff }}$, where $A_{\text {eff }}$ is the effective area of the dipole in the direction of the sun. Since measurements of solar bursts are always made near the maximum of the dipole pattern, $A_{\text {eff }}$ is equal to $A_{0}$ of Eqs. (4) and (7).

Consider first RAD1 at $f \lesssim 1 \mathrm{MHz}$. The method of calibration above is a standard one (e.g. Manning \& Fainberg 1980) that has been used with several other spacecraft, e.g. ISEE-3, Ulysses and Geotail. There is generally good agreement among the independent measurements of flux densities. For example, Nagano et al. (private communication) compared flux densities of identical bursts observed by Geotail and WAVES and found differences smaller than a factor of two between $\approx 0.1$ and $0.8 \mathrm{MHz}$. Furthermore, as seen in Fig. 1, there is good agreement among the measurements of galactic background intensity, and in particular, with the dashed-line extensions of the thick solid line below $0.5 \mathrm{MHz}$ that were derived from RAD1 and from the thermal noise receiver using the above method. However, from about 0.5 to $1 \mathrm{MHz}$ this standard method predicts values of galactic background intensity that are higher at $1 \mathrm{MHz}$ by a factor of about two than are consistent with Eq. (1) or the solid line in Fig. 1; hence, for consistency and to avoid a discontinuity between flux densities from RAD1 and RAD2, we calibrate this range using the galactic background radiation, just as we do for RAD2.

As noted earlier, for RAD2 at frequencies from 1 to 13.8 $\mathrm{MHz}$ there is little experience in space and no other active spacecraft with which to compare, and when the preliminary, pre-launch measurements were first used to calibrate RAD2 there was poor agreement with groundbased observations in the overlapping frequency range of about 7 to $13.8 \mathrm{MHz}$. Therefore we utilize the galactic background radiation as a calibration source as follows:

We use $S_{\text {beam }}^{\text {gal }}$, the thick solid line in Fig. 1 to describe the galactic background. For each antenna, $X, Y$ and $Z$, we require measurements of the receiver noise $P_{\text {rec }}(\nu)$ and 
the galactic background noise $P_{\text {gal }}(\nu)$ at the receiver input, in units of $\mu \mathrm{V}^{2} / \mathrm{Hz}$. For $P_{\text {rec }}(\nu)$, we have spectra taken after WIND was launched but before the antennas were deployed. Then we have measurements of $P_{\text {rec }}(\nu)+P_{\text {gal }}(\nu)$ after the antennas were extended. After subtracting $P_{\text {rec }}(\nu)$ we obtain $P_{\text {gal }}(\nu)$. The power due to the burst, $P_{\mathrm{B}}(\nu)$, is thus:

$$
P_{\mathrm{B}}(\nu)=P_{\mathrm{BRG}}(\nu)-P_{\text {gal }}(\nu)-P_{\text {rec }}(\nu) \quad \mu \mathrm{V}^{2} / \mathrm{Hz}
$$

where $P_{\mathrm{BRG}}(\nu)$ is the measured power of the burst plus receiver noise plus galactic background. The antenna/receiver network term $X(\nu)$ becomes irrelevant because it is common to the observations of the flux density per beam of the galaxy and the flux density of the source.

The conversion from burst power $P_{\mathrm{B}}(\nu)$ in units of $\mu \mathrm{V}^{2} / \mathrm{Hz}$ to flux density $S_{\mathrm{B}}(\nu)$ in physical units is simply:

$S_{B}(\nu)=S_{\text {beam }}^{\text {gal }}(\nu) \frac{P_{\mathrm{B}}(\nu)}{P_{\text {gal }}(\nu)} \quad \mathrm{W} \mathrm{m}^{-2} \mathrm{~Hz}^{-1}$.

At all frequencies above about $0.1 \mathrm{MHz}$, solar burst source sizes are small compared with the beam of a dipole, and they are never at a large angle from the ecliptic plane, so they are near the maximum of the $Z$ antenna pattern, and measurements are made at the maxima of the $X$ or $Y$ antenna patterns as they spin. However, at frequencies lower than about $0.1 \mathrm{MHz}$ the source sizes become very large, $\gtrsim 1$ sr at $f \lesssim 0.1 \mathrm{MHz}$. In the isotropic limit where the source occupies $4 \pi \mathrm{sr}$ (typically about $50 \mathrm{kHz}$ ), its total flux density is underestimated by a factor of $3 / 2$ unless the source size is estimated from the disappearance of spin modulation, and a correction is applied.

\section{Application to a solar type III burst observed from $20 \mathrm{kHz}$ to $47 \mathrm{MHz}$, and conclusion}

The method we have developed to calibrate radio telescopes is useful at frequencies $\lesssim 100 \mathrm{MHz}$ where the galactic background is the major contributor to system noise. It is particularly useful for telescopes of low resolution with which it is difficult to detect discrete radio sources and use them for calibration. Such telescopes are generally used for ground-based observations of the Sun and Jupiter, and for space-based observations of a number of planetary emissions.

The uncertainty in the flux density may be a factor about two. In part, this uncertainty is due to the assumed galactic background spectrum (Sect. 3.1), and in part it is because WAVES and BIRS employ different sampling intervals and frequency resolutions. When observing solar bursts with their rapidly-changing time and frequency structure, these differences can produce differing system responses.

Figure 2 shows the spectrum of a solar type III burst observed from decametric to kilometric wavelengths. Below about $60 \mathrm{kHz}$ the burst was not detectable because its intensity was less than a third that of the receiver noise. The abrupt cutoff of the BIRS spectrum at $6 \mathrm{MHz}$ is due

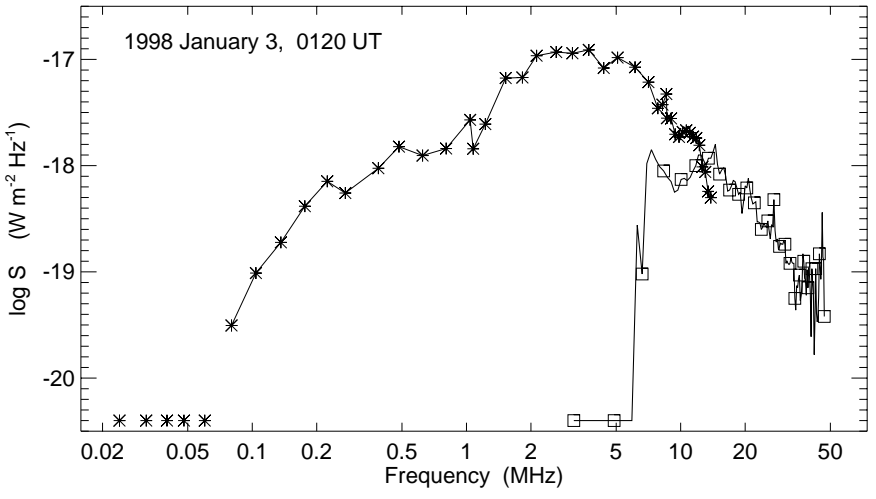

Fig. 2. Spectrum of a solar type III burst that occurred at about 0120 UT on 3 January 1998. The stars and squares mark the spectra observed by WAVES and BIRS respectively. The symbols at the bottom represent non-detections due to a low signal level (stars) or the ionospheric cutoff (squares). The vertical dotted line shows the plasma frequency at $1 \mathrm{AU}$ at the time of the burst

to reflection of the signal by the $\mathrm{F}$ region of the ionosphere, and the flat part from 6 to $12 \mathrm{MHz}$ is affected by absorption as the signal traverses the $\mathrm{D}$ and $\mathrm{E}$ regions. While the correction for absorption described in the text has been applied, it is evidently insufficient, thus indicating a requirement for further analysis of ionospheric effects on signals from the Sun and/or the galactic background.

The spectrum of Fig. 2 is typical of bursts we have studied so far: the flux density increases with frequency to about $10^{-17} \mathrm{~W} \mathrm{~m}^{-2} \mathrm{~Hz}^{-1}$ near $3 \mathrm{MHz}$ and then decreases at higher frequencies. The reason why the maximum in flux density generally occurs near $1 \mathrm{MHz}$ is not known. In some cases it may be due to the following: type III bursts like this one usually start at metric or decametric wavelengths as a group of 10 or more individual bursts, and the electrons of the group then merge into one burst at $1-3 \mathrm{MHz}$, or $4-8$ Ro (e.g. Fig. 3 of Dulk et al. 1998). At this point the beam density is largest with respect to the the background. Further out the beam density decreases faster than the background because of velocity dispersion.

While the spectrum of Fig. 2 is typical of the special class of type III bursts that continue from metric/decametric to kilometric wavelengths, we emphasize that this class represents a small minority of type III bursts. As mentioned in the introduction, many bursts exist only at decametric or shorter wavelengths, while others exist only at long kilometric wavelengths.

However, this special class of solar type III bursts has one exceptional characteristic: on some occasions the flux density near $1 \mathrm{MHz}$ attains values $\gtrsim 10^{-14} \mathrm{~W} \mathrm{~m}^{-2} \mathrm{~Hz}^{-1}$, or $10^{12} \mathrm{Jy}$, or $10^{8} \mathrm{SFU}$ (e.g. Weber 1978), thus surpassing that of any other extraterrestrial source observed in the vicinity of Earth.

Acknowledgements. The WAVES experiment on the Wind spacecraft is a joint project of the Observatory of Paris, the University of Minnesota, NASA/GSFC, and the University 
of Iowa. We thank C. Meetre of NASA (GSFC) for developing the software we used to analyze WAVES observations.

\section{References}

Alexander, J. K., Brown, L. W., Clark, T. A., Stone, R. G., \& Weber, R. R. 1969, ApJL, 157, L163

Bougeret, J.-L., et al. 1995, Space Sci. Rev., 71, 231

Brown, L. W. 1973, ApJ, 180, 359

Cane, H. V. 1977, Non-thermal galactic background radiation, Ph.D. Thesis, University of Tasmania

Cane, H. V. 1978, Aust. J. Phys., 31, 561

Cane, H. V. 1979, MNRAS, 189, 465

Dulk, G. A., Leblanc, Y., Robinson, P. A., Bougeret, J.-L., \& Lin, R. P. 1998, J. Geophys. Res., 103, 17223
Dulk, G. A. 2000, Type III solar radio bursts at long wavelengths, Proc. Chapman Conference on Space-Based Radio Observations at Long Wavelengths, ed. R. Stone, E. Weiler, \& M. Goldstein, Geophys. Monogr., 119, 115

Erickson, W. C. 1997, Publ. Astron. Soc. Aust., 14, 278

Kraus, J. D. 1966, Radio Astronomy (McGraw-Hill), 66 Leblanc, Y., Dulk, G. A., Cairns, I. H., \& Bougeret, J.-L. 2000, J. Geophys. Res., 105, 18215

Manning, R., \& Fainberg, J. 1980, Space Sci. Instr., 5, 161

Novaco, J. C., \& Brown, L. W. 1978, ApJ, 221, 114

Steinberg, J. L., Dulk, G. A., Hoang, S., Lecacheux, A., \& Aubier, M. 1984, A\&A, 140, 39-48

Weber, R. R. 1978, Solar Phys., 59, 377

Yates, K. W., \& Wielebinski, R. 1966, Aust. J. Phys., 19, 389 